\title{
Quantitative Metachromasie mit Pseudoisocyanin: Eine neue Methode zur Bestimmung von Sulfatiden sowie ihre Anwendung bei der Diagnose der Metachromatischen Leukodystrophie (Sulfatid-Lipidose)
}

\author{
Von K. Harzer und H. U. Benz \\ Institut fïr Hirnforscbung (Direktor: Prof. Dr. J. Peiffer) der Universität Tübingen
}

(Eingegangen am 23. Januar/7. Juni 1973)

Es wird eine Methode zur quantitativen Bestimmung von Sulfatiden im Urin beschrieben, bei der die Intensität der ,polymeren Bande“ des metachromasierenden Farbstoffs Pseudoisocyanin gemessen wird. Die Methode ist zum diagnostischen Nachweis der erhöhten Sulfatid-Ausscheidung bei der Metachromatischen Leukodystrophic geeignet. Der bei diescr Erkrankung durch genctischen Enzymdefekt blockierte Sulfatid-Abbau kann mit der Methode ebenfalls nachgewiesen werden. Vor- und Nachteile der Methode sowie die Rolle der Sulfatidmicellen bei der quantitativen Metachromasie werden diskutiert.

\section{Quantitative metachromasia with pseudoisocyanin: $A$ nen method for the determination of sulphatides, and for use in the diagnosis of metacbromatic leucodystrophy (sulphatide lipidosis)}

A method is described for the determination of sulphatides in the urine by measuring the "polymeric band" of the metachromatically active pseudoisocyanin. The determination of the enhanced excretion of sulphatides in metachromatic leucodystrophy is of diagnostic value. The method can be used also to show the enzymatic block in sulphatide degradation in metachromatic leucodystrophy. Problems and advantages of the method as well as the role of sulphatide-micelles in quantitative metachromasia are considered.

Einige Stoffwechselerkrankungen gehen mit der Anhäufung und Ausscheidung von Substanzen einher, die mit bestimmten Farbstoffen eine Metachromasie-Reaktion geben. Zu diesen Krankheiten gehört neben den Mucopolysaccharidosen die Metachromatische Leukodystrophie, bei der Sulfatide im Gewebe angehäuft und im Urin vermehrt ausgeschieden werden. Bei der Metachromasie-Reaktion der Substanzen (also z. B. sauren Mucopolysaccharide und Sulfatide) entstehen mit bestimmten Farbstoffen neue, z. T. gut meßbare Absorptionsbanden. Die Substanzen (bei den Sulfatiden die micellare Form, s. u.) enthalten mehrere negativ geladene Gruppen (Sulfat- oder CarboxylGruppen) in charakteristischer Anordnung (1-4).

Eine Bestimmung der sauren Mucopolysaccharide im Urin mit Pseudoisocyanin als metachromatischem Indikator-Farbstoff ist beschrieben worden (5). Bei der Metachromasie-Reaktion von sauren Mucopolysaccharide mit diesem Farbstoff tritt eine zusätzliche $\mathrm{Ab}$ sorptionsbande bei $573 \mathrm{~nm}$ auf. Diese „polymere Bande" $(2,3,4,6)$ wird durch Aggregation mehrerer Farbstoffmoleküle an den Poiyanionen hervorgerufen.

Im folgenden wird beschrieben, daß bei der Metachromasie-Reaktion von Sulfatiden mit Pseudoisocyanin ebenfalls eine "polymere Bande" entsteht. Sie liegt hier bei $583 \mathrm{~nm}$. Ihre Intensität ist proportional zur Konzentration der Sulfatide. Diese Proportionalität kann für die quantitative Bestimmung der Sulfatide aus Urin ausgenützt werden. Bisher ist keine einfache Methode bekannt, um die Sulfatide im Urin quantitativ zu bestimmen. Meist müssen Dialyseschritte durchgeführt werden, die mit Sulfatidverlust einhergehen. Erfolgt die Bestimmung aus dem Urinsediment, so geht der gelöste Anteil der Sulfatide verloren.

Die Sulfatide gehören zu den Sphingolipiden und werden bei der Metachromatischen Leukodystrophie aufgrund des Defektes der Cerebrosidsulfatase (s. u.) vor allem im Nerven- und Nierengewebe in exzessiver Menge gespeichert. $\mathrm{Da}$ bei der Sulfatidbestimmung die sauren Mucopolysaccharide nicht stören, ist die Methode auch dann anwendbar, wenn sowohl saure Mucopolysaccharide als auch Sulfatide im Urin vermehr ausgeschieden werden, z. B. bei der Austrisschen Variante der Metachromatischen Leukodystrophie (7). Die Methode kann für die Diagnostik der Metachromatischen Leukosdystrophie mit guter Treffsicherheit eingesetzt werden, selbst wenn Enzymansätze zur Bestimmung der Arylsulfatase A (EC 3.1.6.1) nicht durchgeführt werden können (z. B. nach Verlust der Enzymaktivität während des Transports). Die erhöhte Sulfatidausscheidung ist bereits lange vor dem klinischen Stadium der Erkrankung nachweisbar.

Mit der Metachromasie-Reaktion können die Sulfatide auch im enzymatischen Ansatz (z. B. mit Leukocytenenzym) bestimmt werden. Damit ergibt sich eine Möglichkeit, die Sulfatid-spaltende Aktivität (8) der Arylsulfatase A mit einfachen Mitteln durch Verfolgen des Sulfatidumsatzes zu testen.

Für die Sulfatidbestimmung ist Voraussetzung, $\mathrm{da} B$ die Sulfatide in micellarer Form vorliegen, bzw. mindestens zwei Sulfatidmoleküle so zusammengelagert sind, da $B$ 
die Sulfatgruppen den „metachromasie-positiven“ Abstand voneinander haben. Das ist in wäßrigen Lösungen grundsätzlich der Fall, bestimmte Störfaktoren müssen jedoch berücksichtigt werden.

\section{Material und Methodik}

24-h-Sammeluxin wird sofort untersucht oder bei $-20^{\circ} \mathrm{C}$ aufbewahrt. Das 24-h-Urinvolumen sollte innerhalb der altersgemäßen Schwankungsbreite liegen. - Als metachromasierender Farbstoff wird N,N-Diäthyl-pseudoisocyaninchlorid verwendet ${ }^{1}$ ). Der Farbstoff ist bei der Firma Riedel de Haen, Seelze bei Hannover, käuflich. Die Reinheit des käuflichen Farbstoffs ist für die beschriebenen Zwecke völlig ausreichend. In drei dünnschichtchromatographischen Systemen sind im Vergleich zu der ursprünglich benutzten Probe keinerlei Verunreinigungen nachzuweisen. Hinsichtlich der molaren Extinktion und des Schmelzpunktes unterscheiden sich die benutzte Probe und der käufliche Farbstoff kaum (die molare Extinktion ist beim Industrieprodukt um höchstens 10\% geringer; der Schmelzpunkt liegt, nach Sirterung bei $180^{\circ} \mathrm{C}$, mit $252-253^{\circ} \mathrm{C}$ etwas höher als der der benützten Probe mit $243-245^{\circ} \mathrm{C}$ ). Die folgenden benötigten Farbstofflösungen werden jeweils frisch aus einer bei $4^{\circ} \mathrm{C}$ gut haltbaren wäßr. $3 \mathrm{mmol} / 1$ Stammlösung hergestellt. - Die verwendeten synthetischen enzymatischen Substrate ( $p$-Nitrophenylund $p$-Nitrocatechol-Derivate) stammen von der Firma Sigma, St. Louis, USA. - Die Sulfatid- und Cerebrosid-Testsubstanzen sind Präparate der Firma Applied Science Laboratories, Inglewood, USA, und wurden über die Fa. Interchem, München, bezogen. Die gelieferten Chargen der Lipide waren meist von genügender Reinheit, gemäß dünnschichtchromatographischer Analyse. Einmal war die säulenchromatographische Reinigung einer Sulfatid-Charge an Florisil (100-200 mesh, Serva, Heidelberg) erforderlich. - Enzymproben mit Cerebrosidsulfatase-Aktivität wurden aus Gewebs- und Leukocytenextrakten durch isoelektrische Fokussierung präpariert ( $($ ).

\section{Qualitativer Scbnelltest auf Sulfatide im Harn}

Eine gut aufgeschüttelte Urinprobe sowie eine Kontrollurinpool-Probe werden mit 2 Vol. Methanol versetzt. Von dem Gemisch werden $30 \mathrm{ml}$ in einen verschließbaren 100-ml-Meßzylinder gegeben. Mit Chloroform/Methanol $(20 \mathrm{ml}+10 \mathrm{ml})$ wird auf $80 \mathrm{ml}$ aufgefüllt. Das einphasige Gemisch wird dreimal nacheinander mit je $20 \mathrm{ml}$ Wasser phasenverteilt und die jeweils entstehenden Oberphasen werden abgesaugt (die Zwischenschicht und etwa $2 \mathrm{ml}$ Oberphase werden jeweils zurückgelassen). Die letzte Unterphase wird zur Trockene gebracht und in $0,5 \mathrm{ml} 10 \mathrm{~g} / 1$ Natriumcholat- (Serva, Heidelberg) und $1,5 \mathrm{ml} 0,3 \mathrm{mmol} / 1 \mathrm{~N}, \mathrm{~N}$ Diäthyl-pseudoisocyaninchlorid-Lösung aufgenommen. Nach 2 h Stehenlassen und öfterem Umschütteln ist bei erhöhter Sulfatidausscheidung der metachromasiebedingte Stich ins Violette $\left(\mathrm{E}_{583}\right)$ gegenüber der Kontrolle gut erkennbar. Bei sehr geringer Sulfatidausscheidung kann mit doppelten Anfangsvolumina und gleichem Endvolumen gearbeitet werden. - Die erforderlichen Chemikalien sollen in einem Testkit der 'Fa. H. J. Häbich, Hildrizhausen, demnächst zusammengestellt werden.

1) Für die Uberlassung der Farbstoffproben sind wir Prö́f. Dr. G. Scheibe und Dr. J. Suschke, beide Münchẹn, zu außetordentlichem Dank verbunden.

\section{Quantitative Bestimmung der Sulfatide im Urin}

Der zu untersuchende Urin und ein Pool von etwa 20 Kontrollurinen werden auf Raumtemperatur gebracht (nicht eingefroren aufbewahrte Urinproben können falsche Ergebnisse liefern), 1:1 (v:v) mit Methanol verdünnt und kräftig durchgeschüttelt. Es werden Proben von je $10 \mathrm{ml}$ (für die Eichkurve Proben aus dem Kontrollpool, die mit aufsteigenden Sulfatidmengen versetzt sind, z. B. aus einer wäßr. $1 \mathrm{~g} / 1$ Sulfatid-Suspension) mit $0,1 \mathrm{ml} 0,5 \mathrm{~mol} / 1$ Natronlauge versetzt, dann mit $50 \mathrm{ml}$ Chloroform/Methanol $20 \mathrm{ml}+$ $10 \mathrm{ml}$ gemischt, kurz aufgekocht und auf $0^{\circ} \mathrm{C}$ abgekühlt. Jede Fraktion wird kalt in einen Scheidetrichter filtriert und mit $12 \mathrm{ml}$ Wasser phasenverteilt. Nach Klärung der Phasen wird die hydrophobe Unterphase gewonnen und zusammen mit $1 \mathrm{ml} 200 \mathrm{~g} / 1$ Saccharoselösung (durch die Saccharose wird der zu gewinnende Rückstand wasserlöslich) bei etwa $60^{\circ} \mathrm{C}$ zur Trockene gebracht. Der Rückstand wird in $3 \mathrm{ml}$ Wasser unter Erwärmen (etwa $60^{\circ} \mathrm{C}$ ) und Schütteln aufgenommen. Mindestens zwei Aliquote dieser „Suspension $S^{\text {“ }}$ werden mit dem 9 fachen Volumen an $0,15 \mathrm{mmol} / 1$ wäßr. N,N-Diäthyl-pseudoisocyaninchlorid-Lösung versetzt. (Bei nicht vernachlässigbaren Trübungen kann auch mit dem 19 fachen Volumen gearbeitet werden, die Trübungen stören dann nicht mehr, die "Sulfatidspezifische" Extinktion, s. u., ist jedoch noch gut meßbar; der Verdünnungsfaktor muß eingerechnet werden.) - Von den aus Suspension $S$ und $\mathrm{N}, \mathrm{N}$ Diäthyl-pseudoisocyaninchlorid-Lösung bestehenden Fraktionen $F$ wird in einer Küvette mit $2 \mathrm{~cm}$ Lichtweg die Extinktion bei $583 \mathrm{~nm}$ („Sulfatid-spezifische“ Extinktion = „polymere Absorptionsbande“ bzw. Metachromasie der Sulfatide) in der folgenden Weise bestimmt: Zunächst wird der Farbstoff-Leerwert (mit

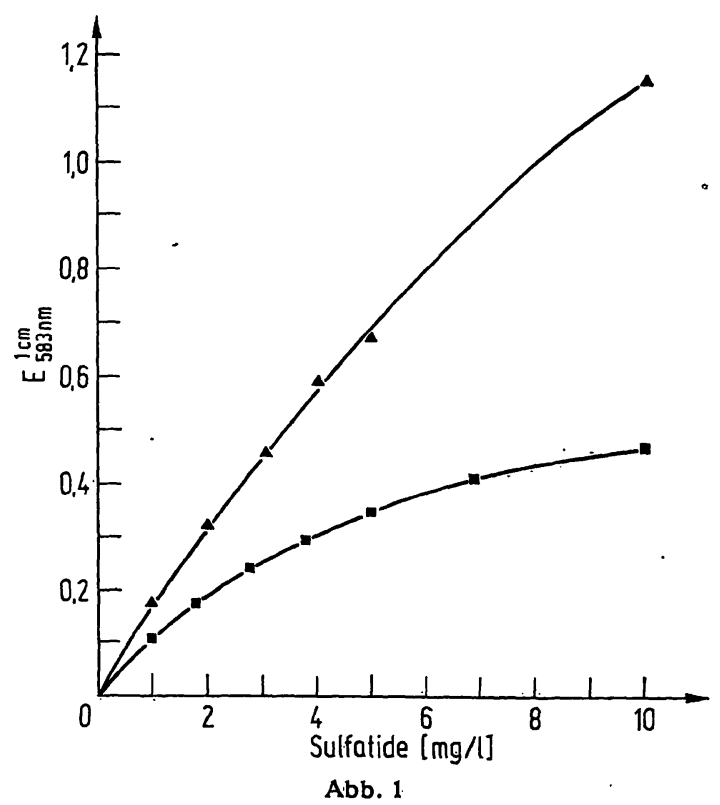

Abhängigkeit der Metachromasie des N,N-Diäthyl-pseudoisocyaninchlorid (,polymere Bande“ bei $\mathrm{E}_{68 \mathrm{~s}}$ ) von der Sulfatidkonzentration: - - wäßrige Lösung, N,N-Diäthyl-pseudoisocyaninchlorid-Konzentration $50 \mu \mathrm{mol} / 1$

wäßrige Lösung mit sulfatidhaltiger Phase des Cerebrosidsulfatase-Ansatzes (s. Methodik), N,N-Diäthyl-pseudoisocyaninchlorid-Konzentration $0,1 \mathrm{mmol} / \mathrm{l}$ 


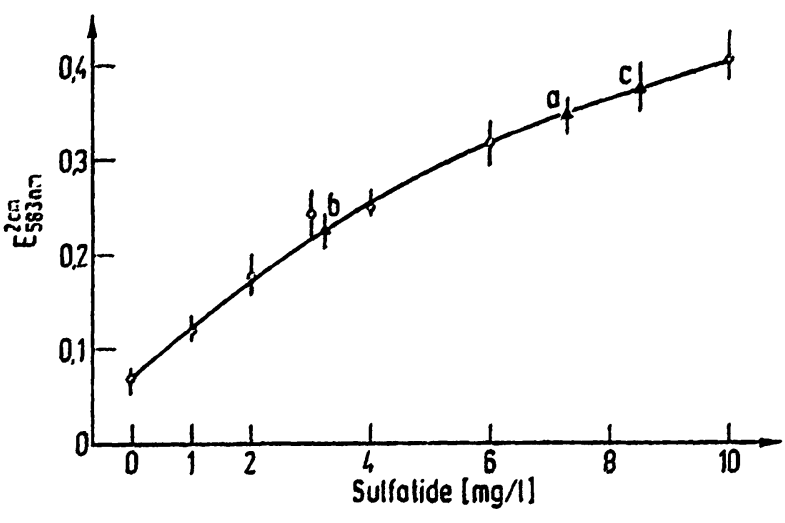

Abb. 2

Abhăngigkeit der Metachromasie des $N, N$-Diäthyl-pseudoisocyaninchlorid (E... von der Sulfatidkonzentration in der hydrophoben Phase chlorid ( $E_{\text {s.8. }}$ ) von der Sulfatidkonzentrat
des Urins

O-O - Pool von 20 Kontrollurinen, mit aufsteigenden Sulfatidmengen versetyt
a) Werte im Urin bel infant

prăklinischen Stadlum

etachromatiacher Leukodystrophie im Werte im Urin bei infantiler Metachromatischer Leukodystrophic im klinisch manifesten Stadlum

c) Werte im Urin bel adulter Metachromatischer Leukodystrophie

Dic angegebenen Schwankungen entsprechen Dreifachbestimmungen desselben Urins

Wasser anstatt Suspension S) bei $583 \mathrm{~nm}$ auf 0 gestellt. Nun wird bei sonst gleicher Einstellung die Wcllenlänge nach $562,5 \mathrm{~nm}$ (Bezugswcllenlänge, bei der im wesentlichen nur der monomere Farbstoff absorbiert) verschoben und die Lecrwert-Extinktion $\mathrm{E}_{\text {ret }}$ (\%. B. 0,90) abgelesen. Jet\%t wird die erste der Fraktionen $\mathrm{F}$ in die Küvette gegeben und bei $562,5 \mathrm{~nm}$ auf den Wert $\mathbb{E}_{\text {ret }}$ gestellt. Dann wird dic Wellenlänge wicder nach $583 \mathrm{~nm}$ verschoben und die „Sulfatidspezifische" Extinktion abgelescn. Als nächstes folgt wieder Lecrwert, dann Fraktion $F$ und so weiter. Mit den Werten für $\mathrm{E}_{583}$ wird in dic analog erhaltenc Eichkurve (Abb. 2) eingegangen und der Sulfatidgchalt des Urins abgclesen. Im Normalfall licgt $\mathbb{B}_{5 \% 3}^{2 e m}, 2 \mathrm{nj}^{\prime} \mathrm{C}$ um etwa 0,05 , bei exhöhter Sulfatidausscheidung meist über etwa 0,15. - Nach Aufnchmen der getrockneten Unterphase und Zugabe von N,N-Diäthyl-pscudoisocyaninchlorid-Lösung sollte das Ablesen der Extinktionen innerhalb einer Stunde erfolgen (spätcr nimmt dic Extinktion langsam ab). Auf Temperaturkonstan\% ist $r x$ achten. Bei-tieferen Temperaturen $\left(\%\right.$ B. $\left.+4^{\circ} \mathrm{C}\right)$ werden höhere Extinktionen crhalten.

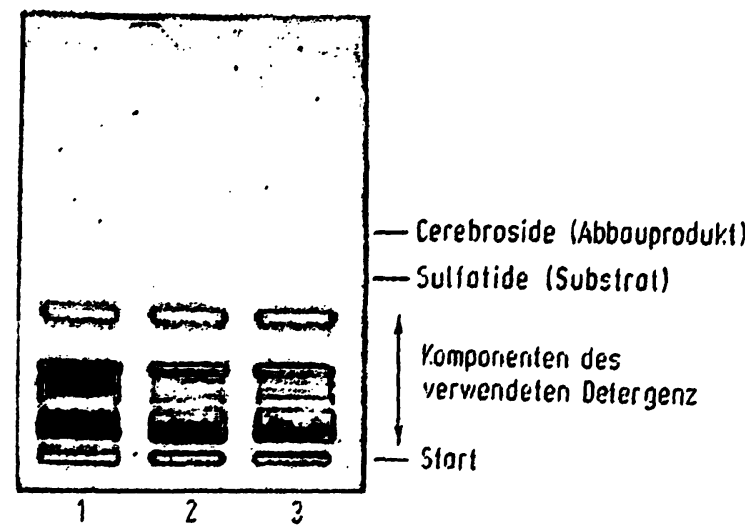

Ady. 3

Dannschichtchromatographizcher Nachweig der Cerebrosidsulfatase Aktivităt. Es wurden $\int e 40 \mu l$ der $48 \mathrm{~h}$ inkuhierten Enzymansät\%e auf getragen. Fliefmittel, Anfärbung usw. s. Methrodik

Bahn 1: Enzym aus normaler Rectumbiopsic gewonnen (unveriffentl. Ergebnisse) Bahn 2: Enzym aus leukocyten bei Metachromatischer Leukody-

Bahn 3: Enzym aus Kontroll-Leukecyten kewonnen

Aut Bahn 2 (Metachromatischer Leukodystrophic) sind keine Cerebroside entstanden

Zur. Bestätigung der Verclachtsdiagnose „Mctachromatische Leukodystrophic" kann auf cinfache Art dic Arylsulfatase $\Lambda$ im Urin bestimmt werden: 3 - bis 5stündige Inkubation von gleichen 'Teilen dialysierten Urins und der $p$-Nitrocatecholsulfat-Substratlösung nach Batsm et al. (12); Bc\%ug dcr. Bn\%ymaktivität auf dic analog bestimmten Aktivitäten der $\beta$-Hexosaminidase (EC 3.2.1.30) und der sauren Phosphatase (EC 3.1.3.2). Ist dic Arylsulfatase $A$ im Urin stark crniedrigt, bestimmen wir das En\%ym auch noch in den Jeukocyten (9). Noch sperifischer ist dic Bestimmung der Cerebrosidsulfatase in den Jecukocyten.

Messung der C'crebrosidsulfatase-Aktivität durch Sulfalidbestimmung mil $N, N$-Diälbyl-pseudoisocyaninchlorid im enzymalischen Ansal\%.

Folgende Detergen\%- und Substrat-J,ösungen in Chloroform/Methanol $20 \mathrm{ml}+10 \mathrm{ml}$ werden vercinigt und zur Trockene gebracht:

1. $10 \mathrm{~g} / \mathrm{l}$ "rohcs Natriumtaurocholat" (10) (Schuchardt, Münchon, Kat.-Nr. 'ГA 039)

2. $1,1 \mathrm{mmol} / 1$ Sulfatide, und zwar $0,8 \mathrm{ml}$ bei Cerebrosidsulfatase-Proben aus Gewche mit cincr Aryl-

Tab. 1

Anderung der N,N-Dläthyl-pseudoisocyaninchlorid-Metachromasie bzw. des Sulfatidgehalts in dor lyydrophoben Phase des CerchrosidsulfataseAnsatzes. Die Schwankungen entsprechen Dreifachbestimmungen aus demsell,en Enzymansat\%. Mi, () . Mctachromatische Leukodystrophie

\begin{tabular}{|c|c|c|c|c|c|}
\hline \multirow[t]{2}{*}{$\begin{array}{c}\text { Inkubationszeit } \\
\text { (h) }\end{array}$} & & \multirow{2}{*}{$\begin{array}{c}\text { Ebsgn } \\
\text { Enzymquelle } \\
\text { Leukocyten }\end{array}$} & \multirow[b]{2}{*}{$\begin{array}{l}\text { Leukocyten } \\
\text { (MLID) }\end{array}$} & \multicolumn{2}{|c|}{$\begin{array}{l}\% \text { Sulfatidabbau } \\
\text { Enzymquelle }\end{array}$} \\
\hline & Nicre & & & Nicre & leukocyten \\
\hline 0 & $\begin{array}{c}0,37 \\
(0,32-0,40)\end{array}$ & $\begin{array}{c}0,10 \\
(0,15-0,24)\end{array}$ & $\begin{array}{c}0,20 \\
(0,18-(), 23)\end{array}$ & () & 0 \\
\hline 4 & $\begin{array}{c}0,34 \\
(0,31-0,38)\end{array}$ & & & 12 & \\
\hline 8 & $\begin{array}{c}0,32 \\
(0,28-0,33)\end{array}$ & & & 22 & \\
\hline 18 & $\begin{array}{c}0,23 \\
(0,18-0,27)\end{array}$ & & & 52 & \\
\hline 60 & & $\begin{array}{c}0,14 \\
(0,10-0,10)\end{array}$ & $\begin{array}{c}0,22 \\
(0,18-0,25)\end{array}$ & & 27 \\
\hline
\end{tabular}


sulfatase-A-Aktivität von etwa $100-300 \mathrm{nmol}$ gespaltenem $p$-Nitrocatechol-Sulfat je min je mg Protein (9) bei $37^{\circ} \mathrm{C}$; bzw. $0,4 \mathrm{ml}$ bei Enzymproben aus Leukocyten mit einer Arylsulfatase-A-Aktivität von etwa $50 \mathrm{nmol} \mathrm{je} \mathrm{min} \mathrm{je} \mathrm{mg} \mathrm{Protein.}$

Der Trockenrückstand wird in $2,0 \mathrm{ml}$ Enzymprobe $(1,2-6,0 \mathrm{mg}$ Protein) suspendiert und mit $0,2 \mathrm{ml}$ Natriumacetat-Puffer $(0,5 \mathrm{~mol} / \mathrm{l}, \mathrm{pH} 5,0)$ sowie $80 \mu \mathrm{l}$ einer wäßr. $1 \mathrm{~g} / 1$ Lösung von Ampicillin (Binotal, Bayer) versetzt. Je zwei Proben zu 0,2 $\mathrm{ml}$ der Suspension werden nach Inkubationszeiten von $0,15,30$ und $60 \mathrm{~h}$ bei $37^{\circ} \mathrm{C}$ entnommen. Jede Probe wird mit $50 \mathrm{ml}$ Chloroform/Methanol $20 \mathrm{ml}+10 \mathrm{ml}$ versetzt und bei $-20^{\circ} \mathrm{C}$ aufbewahrt. Jede dieser Fraktionen wird kalt in einen Scheidetrichter filtriert und mit $10 \mathrm{ml}$ Wasser verteilt.

Nach völliger Klärung wird die Unterphase gewonnen und zusammen mit $1 \mathrm{ml} 200 \mathrm{~g} / \mathrm{l}$ Saccharoselösung bei $60^{\circ} \mathrm{C}$ zur Trockene gebracht. Der Rückstand wird in $3 \mathrm{ml}$ einer wäßr. Lösung von $0,1 \mathrm{mmol} / \mathrm{l} \mathrm{N}, \mathrm{N}$-Diäthylpseudoisocyaninchlorid aufgenommen. Die Extinktion bei $583 \mathrm{~nm}(1 \mathrm{~cm})$ wird abgelesen (hinsichtlich einzuhaltender Zeiten und Temperaturkonstanz siehe vorhergehenden' 'Abschnitt). Die Exktinktionsabnahme nach den verschiedenen Inkubationszeiten ist ein $\mathrm{MaB}$ für die Cerebrosidsulfatase-Aktivität (Tab. 1, Eichkurve in Abb. 1 unten).

\section{Dünnschichtcbromatographischer Nacbweis der Cerebrosid- sulfatase-Aktivität}

Zur Kontrolle der Cerebrosidsulfatase-Bestimmung mit N,N-Diäthyl-pseudoisocyaninchlorid werden $40 \mu \mathrm{l}$ des Enzymansatzes (Suspension, s. vorhergehenden Abschnitt) zum Zeitpunkt 0 und nach Inkubationszeiten von 12 bis $96 \mathrm{~h}$ (bei $37^{\circ} \mathrm{C}$ ) auf Dünnschichtplatten (Kieselgel-G-Fertigplatten, Merck, Darmstadt, Art.Nr. 5729) aufgetragen und im Fließmittel Chloroform/ Methanol/konz. Ammoniaklösung $70 \mathrm{ml}+30 \mathrm{ml}+$ $1 \mathrm{ml}$ bis zur Fronthöhe $15 \mathrm{~cm}$ chromatographiert. Nach Trocknen, Ansprühen mit KäGI-MIESCHER-Reagenz $(1 \mathrm{Vol} 20 \mathrm{~g} / 1$ Schwefelsäure $+1 \mathrm{Vol} 6 \mathrm{~g} / 1$ Anisaldehyd in Eisessig) und $10 \mathrm{~min}$ Erhitzen auf $130^{\circ} \mathrm{C}$ werden die mit Hilfe von Testsubstanzen (s. oben) charakterisierten Sulfatid- und Cerebrosid (= Abbauprodukt)Flecke sichtbar.

\section{Diskussion}

Voraussetzung für die Bestimmung von Sulfatiden im Urin durch quantitative Metachromasie ist, da $B$ auch unter pathologischen Bedingungen keine wesentlichen Mengen anderer metachromatischer Substanzen (außer sauren Mucopolysacchariden, die nicht stören) durch die Niere ausgeschieden werden.

Die Sulfatide erzeugen in wäßriger „Lösung “ - ebenso wie im histologischen Schnitt (11), wo eine dichte Packung der Moleküle vorliegt - eine ähnliche Metachromasie wie saure Mucopolysaccharide. Die Sulfatide dürften also in den untersuchten Konzentrationen mindestens teilweise Micellen bilden, so daß die Sulfat- gruppen einen definierten, „metachromasie-positiven “ Abstand voneinander haben. Die Sulfatide ergeben in den benutzten Konzentrationen optisch klare wäßrige Lösungen. Werden diese Lösungen aus Sulfatiden hergestellt, die zuvor aus organischen Lösungsmitteln zur Trockene gebracht waren, so erreichen sie erst nach einiger Zeit ihre volle metachromatische Eigenschaft. Die dissoziierten Molekülé lagern sich im Wasser wohl langsam wieder zu Micellen zusammen.

Die „polymere Bande" (s. oben) liegt nach unsern Ergebnissen für Sulfatide bei $583 \mathrm{~nm}$, für saure Mucopolysaccharide liegt sie bei $573 \mathrm{~nm}$ (s. Einleitung). Die Ursache dieser Verschiebung der Wellenlänge wird untersucht (SCHEIBE, persönliche Mitteilung). Eine Begründung kann vorläufig nocht nicht gegeben werden. Man kann spekulieren, daß die metachromasiepositive Sulfatid-Micelle Kugelform bestitzt, an die sich die N,N-Diäthyl-pseudoisocyaninchlorid-Moleküle mit etwas anderem Abstand voneinander anlagern als an das metachromasie-positive saure MucopolysaccharidMolekül mit seiner Kettenform, so daß die „polymeren Absorptionsbanden" für Sulfatide und saure Mucopolysaccharide bei verschiedenen Wellenlängen liegen. Die beiden Optima bei 583 und $573 \mathrm{~nm}$ lassen sich unter bestimmten, an anderer Stelle zu erläuternden Bedingungen, nebeneinander darstellen.

Für eine bestimmte Sulfatidkonzentration läßt sich eine Sättigungskurve für den Grad der Metachromasie $\left(E_{583}\right)$ bei Erhöhung der Farbstoff konzentration verfolgen. Sättigung ist erreicht bei etwa $1 \mu \mathrm{mol} \mathrm{N}, \mathrm{N}-$ Diäthyl-pseudoisocyaninchlorid je $1 \mu \mathrm{mol}$ Sulfatide. Das entspricht einem molaren Verhältnis von 1:1. Die Metachromasie-Reaktion erfolgt also offenbar stöchiometrisch. Oberhalb der Sättigungskonzentration besteht eine gute Proportionalität (Abb. 1 obere Kurve) zwischen Sulfatidkonzentration und $\mathrm{E}_{588}$. Für die beschriebene Bestimmungsmethode werden N,N-Diäthylpseudoisocyaninchlorid-Konzentrationen verwendet, die weit über der Sättigungskonzentration liegen, um bei der Metachromasie-Reaktion störende Komponenten der untersuchten Fraktionen zurückzudrängen. Werden die Sulfatide - aufgrund ihres metachromatischen Effekts in wäßrigen Lösungen bestimmt, die noch zahlreiche andere Komponenten enthalten, also z. B. in Urin oder enzymatischen Ansätzen, so ist mit Störungen zu rechnen. Nach Phasenverteilung von Urin und enzymatischen Ansätzen (s. unten) ist jedoch eine gut meßbare Beziehung zwischen Sulfatidgehalt und Metachromasie $\left(E_{583}\right)$ festzustellen. Die Eichkurven verlaufen allerdings nicht ganz so günstig wie bei Verwendung reiner Sulfatidlösungen (vgl. Kurve in Abbildung 1 oben mit den Kurven in Abbildung 1 unten und in Abbildung 2).

Die Sulfatidbestimmung mit N,N-Diäthyl-pseudoisocyaninchlorid aus der hydrophoben Phase des Urins erfaßt über $90 \%$ der Sulfatide. Beträchtliche Mengen von sauren Mucopolysacchariden, wie sie z. B. bei der Austrnschen Variante der Metachromatischen Leukodystrophie (7) ausgeschieden werden, stören nicht. Zur 
Diagnostik der Metachromatischen Leukodystrophie wird meist die Bestimmung der Arylsulfatase A herangezogen; die beschriebene Sulfatidbestimmung im Urin ist jedoch fast ebenso zuverlässig. Sie liefert auch dann noch eindeutige Ergebnisse, wenn die enzymatische Aktivität im Urin nicht sicher beurteilt werden kann. Die Sulfatidausscheidung bei Metachromatischer Leukodystrophie im Urin schwankt naturgemäß, ist jedoch immer nachweisbar. Bei 4 Fällen wurden Werte zwischen 5 und $16 \mathrm{mg}$ je 1 Urin gefunden. Schon im präklinischen Stadium der Erkrankung (Abb. 2, Metachromatische Leukodystrophie a, Alter des Pat. 6 Mon.) wurde ein Wert von $7 \mathrm{mg} / 1$ Urin festgestellt ${ }^{1}$ ). Bei der enzymatischen Diagnostik der Metachromatischen Leukodystrophie kann die Bestimmung der Cerebrosidsulfatase den Test auf Arylsulfatase A unter Umständen sinnvoll ergänzen: Die Bestimmung der Cerebrosidsulfatase ist noch spezifischer (vgl. 8, 9), da die Spaltung des natürlichen, im Krankheitsfalle als Speicherstoff angehäuften Substrats gemessen wird. Der Test der Arylsulfatase A ist zwar einfacher, die Cerebrosidsulfatase läßt sich mit Hilfe von N,N-Diäthylpseudoisocyaninchlorid jedoch auch mit relativ geringem Aufwand testen.

Die Cerebrosidsulfatase ist unter den angegebenen Bedingungen nicht vollständig mit Substrat gesättigt

1) Aus postmortalen frischen oder formalinfixierten Geweben (Hirn oder Niere) gelingt es ebenfalls, die Metachromatische Leukodystrophie mit Hilfe von N,N-Diäthylpseudoisocyaninchlorid recht sicher zu diagnostizieren. Für den Schnelltest wird eine kleine Menge eines $50 \mathrm{~g} / \mathrm{l}$ Chloroform/Methanol $(20 \mathrm{ml}+$ $10 \mathrm{ml}$ )-Extraktes getrocknet und der Rückstand in demselben Volumen an $0,3 \mathrm{mmol} / 1$ N,N-DiäthylpseudoisocyaninchloridLösung unter Erwärmen aufgeschwemmt; nach Abkühlen zeigt sich die Metachromasie im Vergleich zum Normalgewebe deutlich. (vgl. (9), $\mathrm{K}_{\mathrm{m}}$ etwa $\left.0,4 \mathrm{mmol} / \mathrm{l}\right)$. Bei Sättigung würden zwar noch höhere Sulfatidumsätze erzielt, aber die Abnahme von $\mathrm{E}_{583}$ wäre weniger genau meßbar (flacher Bereich der Eichkurve in Abb. 1 unten). Bei den untersuchten Fällen von Metachromatischer Leukodystrophie konnte keine Abnahme festgestellt werden ( $z$. B. Tab. 1).

Da mit sehr geringen Konzentrationen von Sulfatiden $(1 \mu \mathrm{mol} / \mathrm{l})$ in den enzymatischen Ansätzen bereits Metachromasie exzeugt werden kann, wozu die Aggregation von mindestens zwei Molekülen notwendig ist (deren Sulfatgruppen haben dann den "metachromasie-positiven" Abstand), greift die Cerebrosidsulfatase ihr Substrat möglicherweise nur (oder auch) dann an, wenn es in di- oder oligomerer Form vorliegt. Die quantitative Metachromasie in wäßriger Lösung ist unseres Erachtens außer bei den geschilderten diagnostischen und enzymatischen Fragen auch bei mehr grundsätzlichen Problemen ein gutes Hilfsmittel, so z. B. bei der Abschätzung der kritischen micellaren Konzentration der Sulfatide (und evtl. vergleichbarer Substanzen). Auch die Kinetik der Detergentien-Einwirkung auf metachromasierende Komplexe kann verfolgt werden, wobei sich z. B. zeigt, daß Komplexe mit Sulfatid wesentlich schneller dissoziieren als solche mit sauren Mucopolysacchariden, wohl infolge der Auftrennung der Sulfatidmicellen.

\section{Danksagung}

Fräulein INGRID ScHUSTER möchten wir für ihre ausgezeichnete technische Hilfe danken.

Die Arbeit wurde durch eine Sachbeihilfe der Deutschen Forschungsgemeinschaft unterstützt.

\section{Literatur}

1. Harms, H. (1965), Handbuch der Farbstoffe für die Mikroskopie, Staufen-Verlag Paul Bercker, Kamp-Lintfort S. I/86I/111. - 2. Schauer, A. \& Scheibe, G. (1959), Histochemie, 1, 190-195. - 3. SCHUEBLER, T. H. (1958), Naturwissenschaften 45,214 . - 4. SCHIEBlER, T. H. \& SchiessLER, S. (1959), Histochemie 1, 445-465. - 5. SUSCHKE, J., VoGt, D. \& SCHETBE, P. (1967), Fortschr. Med. 85, 911-912. 6. Appex, W. \& Scherbe, G. (1958), Z. Naturforschung B 13, 359-364. - 7. Austin, J., Armstrong, D. \& Shedrer, L.
(1965), Arch. Neurol. 13, 593-614. - 8. MeHL, E. \& JATZKEwrtz, H. (1965), Biochem. Biophys. Res. Commun. 19, 407-411. 9. Harzer, K., Stinshoff, K., Mraz, W. \& JatzKewitz, $H$. (1973), J. Neurochem. 20, 279-287. - 10. SANDHOFf, K., Harzer, K., Wässle, W. \& Jatzkewitz, H. (1971), J. Neurochem. 18, 2469-2489. - 11. v. Hirsch, T. \& Peiffer, J. (1955), Arch. Psychiat. Nervenkr. 194, 88-104. - 12. BAUM, H., Dodgson, K. S. \& Spencer, B. (1959), Clin. Chim. Acta 4, $453-455$ 